\title{
On the Context-aware, Personalized Delivery of Process Information: Viewpoints, Problems, and Requirements*
}

\author{
Markus Hipp*, Bela Mutschler ${ }^{\dagger}$, Manfred Reichert ${ }^{\ddagger}$ \\ ${ }^{*}$ Group Research \& Advanced Engineering, Daimler AG, Ulm, Germany \\ markus.hipp@daimler.com \\ $\dagger$ University of Applied Sciences Ravensburg-Weingarten, Germany \\ bela.mutschler@hs-weingarten.de \\ $\ddagger$ Institute of Databases and Information Systems, University of Ulm, Germany \\ manfred.reichert@uni-ulm.de
}

\begin{abstract}
Enterprises are confronted with an increasing amount of data. This data overload makes it difficult to provide knowledge-workers and decision-makers with the needed information. Particularly challenging in this context is the integrated provision of both structured and unstructured information depending on the current process context and user, i.e., the context-aware, personalized delivery of process information. Examples of unstructured process information include all kinds of office documents or e-mails. Examples of structured process information are business process models or data from enterprise information systems. Picking up the need for a context-aware, personalized delivery of process information, this paper presents results from three empirical studies: two exploratory case studies from the automotive domain and the healthcare sector, and an online survey among 219 participants. In a first step, we identify and describe problems with respect to process-oriented information management in general and the personalized provision of process information in particular. In a second step, we derive requirements on the user-adequate handling of process information.
\end{abstract}

Keywords-process information; end-user; empirical research

\section{INTRODUCTION}

Globalization results in significant competitive pressure for enterprises. In particular, new forms of (crossorganizational) collaboration and knowledge-intense business processes need to be adequately supported. As examples consider distributed engineering processes in the automotive industry [1] and the treatment of patients in integrated healthcare networks [2]. To meet this challenge, enterprises have to implement an effective business process management (BPM) [3] based on tools for process modelling [4], process execution [5][6], and process analysis [7]. Consequently, related research fields have gained significant attention in recent years. By contrast, what has been neglected so far, is the support of knowledge-workers and

\footnotetext{
*This research was done in the niPRO project which is funded by the German Federal Ministry of Education and Research (BMBF) under grant number $17102 X 10$. More information can be found at http://www.niproproject.org.
}

decision-makers by delivering personalized process information depending on the users' current process context.

Thereby, the integrated provision of both structured and unstructured process information is particularly challenging. Structured process information can be characterized as information that can be automatically processed by an IT system, and can thus be automatically related to other information. Unstructured information, in turn, can only be interpreted by humans, as it does not contain any contextual references. As examples of structured process information consider graphical business process models or data from enterprise information systems such as ERP or CRM systems. Examples of unstructured process information include all kinds of office documents or e-mails, including mainly plain text. In this paper, process information particularly refers to a specific process step, or, in other words, to information being relevant for a user in a specific process context.

However, providing process information at the right time and in the right format and granularity is far from being trivial [8]. Today, traditional information management concepts [9] and information retrieval approaches [10] are used for this task. Office documents are provided using shared drives, appointments are managed using personal information management tools, e-mails are analyzed using full text search engines, and business data is provided by enterprise information systems. However, process information still has to be manually linked to a given process context. This results not only in significant efforts for maintaining these links, but also hampers effective information processing by users. As an example consider the healthcare sector. Medical records often comprise hundreds of (handwritten) notes and sheets. This makes it difficult to maintain informational overview, especially since much information are "hard-wired" with certain steps of the patient treatment process.

What is needed in practice is process-centric information logistics. However, due to the complexity of many business processes and the variety of process information being relevant in practice, user needs cannot be satisfied by applying existing information logistics approaches, which 
often neglect the end-user perspective. Picking up this issue and providing empirical evidence for it, this paper presents results from three empirical studies: two exploratory case studies from the automotive domain and the healthcare sector, and an online survey among 219 participants. In a first step, we identify and describe problems with respect to process-centric information logistics in general and the integrated provision of both structured and unstructured process information in particular. Thereby we adopt a strict end-user perspective. In a second step, we derive requirements on the user-adequate handling of process information. Specifically, we investigate the following research question:

\section{What are requirements on the handling of} process information from a user perspective?

The remainder of this paper is organized as follows. Section II gives background information. Section III presents the results of the two case studies. Section IV summarizes the results from the online survey. Section V discusses related work. Finally, Section VI concludes the paper and gives an outlook.

\section{BACKGROUND INFORMATION}

\section{A. Process Information Portals}

The presented empirical research has been performed in the niPRO project. In this project we apply semantic technology to integrate process information in intelligent, personalized process information portals. Overall goal is to support knowledge-workers and decision-makers with the needed process information depending on their preferences and current process context.

The used semantic technology enables the construction of semantic information networks. Such networks represent information, relationships, relation reasons (RR) and relation weights (RW), and enable the automated analysis and context-aware selection of required (process) information [11]. A specific process context corresponds to a clipping of the overall information network. As example consider the semantic information network depicted in Figure 1. Starting from an arbitrary information object ("task" in our example; e.g., the planning of a new project), related information objects (in our example: lessons learned, customers, persons associated with "task") can be automatically recognized as information objects being relevant for the current process context. Note that each "node" (i.e., information object) can be the starting point for the analysis and selection of process information.

The niPRO process information portal aims at determining required information for knowledge-workers and decisionmakers dynamically and automatically. Key challenges include, the role-oriented provision of process information, the flexible visualization of process information and the management of different levels of information granularity. The niPRO project goals can be summarized as follows:

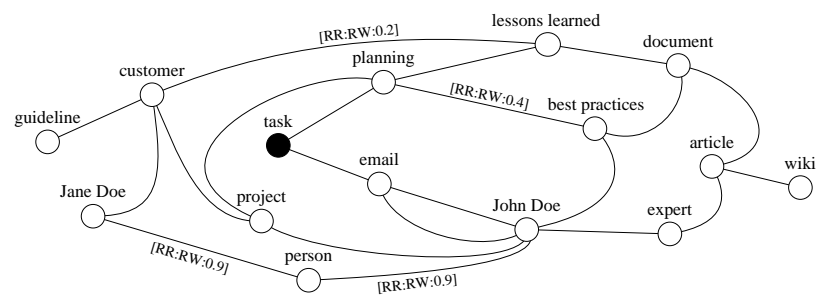

Figure 1. Example of a semantic information network.

- Context-aware retrieval and provision of relevant process information

- Group-oriented, role-based access on process information (personalization)

- Integration of both structured and unstructured process information

- Semantic analysis and linking of process information

Thereby, niPRO will follow a strict user-centered design approach [12], i.e., end users are involved in the entire research process to ensure that a user-adequate solution is designed based on the requirements of the process participants. niPRO will be applied to complex engineering processes for electric/ electronic components in the automotive industry. Additional application scenarios stem from the healthcare sector.

\section{B. Process Information}

Generally, we have to distinguish between data, information, and knowledge. Ahsan and Shah [13] state that data is raw and can be commonly seen as simple facts. Further, they can be represented in various forms and do not have a meaning. For example, a patient has a "temperature". Information, in turn, constitutes data that gets a meaning through interpretation [14]. Regarding the aforementioned healthcare scenario, information can help doctors when treating the patient. As example consider a patient whose temperature has risen by two degrees within the last two days. This information can be useful when diagnosing the patient. Finally, knowledge can be considered as evidencebased information being interpreted and put into a specific context. For example, if a patient shows other symptoms in addition to a rising temperature, the doctor can conclude that the patient caught a flu.

In the following, process information refers to a specific process step, i.e, to information being relevant for a user in a specific process context. Thereby, we have to additionally distinguish between process schema and process instance. So far, both research and practice have not addressed in sufficient detail how processes and process information can be effectively merged. Currently, either fixed synchronization points (hard-wiring process and process information) or conventional information retrieval and enterprise search engines are used for this purpose. 


\section{EMPIRICAL ANALYSIS}

This section presents results of the two case studies we performed to answer the research question from Section I. Section III-A sketches the research methodology we used to derive requirements from the case studies. Section III-B presents the first case study at a large clinic. Section III-C summarizes the second case study from the automotive domain. Section III-D discusses our findings.

\section{A. Research Methodology}

The main goal of our case study interviews is to identify problem areas (PA) hampering the effective handling of process information from the end-user perspective. Each PA is analyzed based on three viewpoints (cf. Fig. 2). This becomes necessary as the handling of process information by a particular user is not only influenced by his personal preferences and procedures, but also by other, more general aspects:

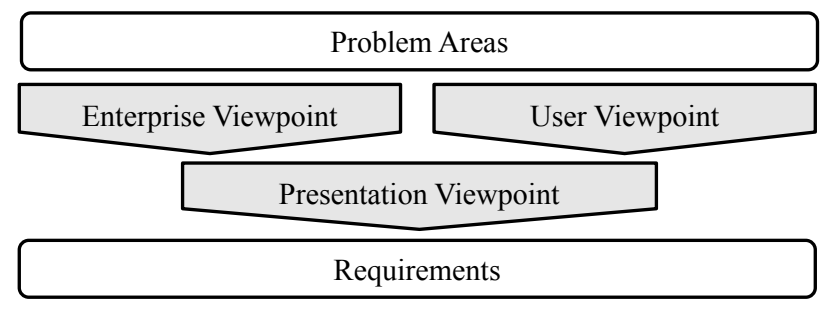

Figure 2. Our case study approach.

- The enterprise viewpoint deals with problems and challenges emerging from general organizational characteristics. Examples of such characteristics include management requirements (e.g., on the documentation of processes), organizational structures (e.g., departments or business units), governance rules (e.g., on the access to process information), and compliance rules (e.g., on the protection and archiving of process information). Note that problems associated with this viewpoint affect all users.

- We also investigate each PA from the perspective of individual users (we call this the user viewpoint). Specifically, we are interested in issues which complicate or even prevent the effective and efficient handling of process information due to typical preferences and procedures of single users. As a first example consider the phenomenon that employees generate shadow copies of documents being important for their own work on private drives. Also consider the lack of an overview on the overall progress of a process due to limited access of users to process information.

- Finally, we adopt a presentation viewpoint, which addresses problems emerging from the tool-based presentation, provision and visualization of process information. Findings associated with this viewpoint can be considered as a direct consequence of the problems associated with the first two viewpoints.

\section{B. Case Study 1: Clinical Sector}

The first case study took place in a large hospital in Southern Germany. Eight interviews were performed in five different departments. The sequence of the interviews followed a characteristic patient treatment process from patient admission to surgery to invoicing. Doing so, we were able to involve most relevant stakeholder (doctors, nurses, administrative staff etc.) and therewith typical users of process information.

In this case study we identified three PAs:

\section{1) Problem Area 1 - Tasks:}

Enterprise Viewpoint: Participants state that many tasks (and entire processes) are not properly defined. As a task may be performed by different departments, this can result in varying quality levels concerning task execution. As example consider patient admission. This task is generally performed by the department for admission. However, in emergency cases, patients may be also admitted by nurses in a ward. Besides, we identified other critical issues concerning the processing of tasks. For example, certain tasks are solely performed by experienced clinical staff. Main reason is that guidelines for tasks mainly exist in paper form and are thus hard to find (e.g., in cabinets). Also, interview participants complained about lack of time to study existing guidelines. Moreover, actuality of guidelines is often not given as distributed copies exist throughout the hospital. Finally, participants stated that keeping an overview of documents' actuality is nearly impossible. The main requirement from the enterprise viewpoint: To ensure a high quality of task results guidelines for tasks have to be defined at an appropriate level of granularity (R1).

User Viewpoint: For a single employee task results are often more important than the actual execution of the task. As example consider again patient admission. One outcome of this task is the delivery of information on the patient's health insurance. For example, this information is indispensable (with few exceptions) when planning a surgery. Interview participants also stated that task results are mainly exchanged in paper form (e.g., surgery reports or findings) or orally (e.g., via phone). For example, the nurse has to manually transfer the documents resulting from a patient admission to the department for admission, where all patient information is centrally managed.

The most important requirements from the user viewpoint are as follows: To standardize task results, inputs and outputs of single tasks need to be well defined (R2) (e.g., in the form of documents). Also, the exchange of task results should be automatically enforced (R3) to guarantee a seamless processing of the process and to avoid information loss. 
Presentation Viewpoint: From the presentation viewpoint we can pick up R1 and conclude that the first presentation requirement is that guidelines for tasks must be visualized in an appropriate manner (PR1), i.e., information on tasks must be easy to find and comprehend. Regarding $\mathrm{R} 2$ and R3 the enforcement of information exchange should be graphically visualized (PR2), e.g., providing step-by-step guidance using workflow technology.

\section{2) Problem Area 2 - Patient Treatment Process:}

Enterprise Viewpoint: From the enterprise viewpoint a major problem is the missing documentation of general patient treatment processes. In particular, medical departments are often unaware of the current process step. This, in turn, hampers a seamless execution of patient treatment processes when crossing departmental borders. Consequently, the most important requirement from the enterprise viewpoint is that hospital-wide, documented treatment processes being easily accessible in every department are required ( $\mathrm{R} 4)$.

User Viewpoint: Participants also stated that communication between departments is sub-optimal. This becomes particularly important when temporal constraints have to be taken into account. Think of a notification at the ward when a patient has to be transferred back to that ward from the operating theatre. Hence, the most important requirement from the user viewpoint is to continuously provide information on the process progress (R5).

Presentation Viewpoint: Concerning the presentation viewpoint, a graphical visualization of the entire patient treatment process (PR3) is required (which follows from R4 and R5). In particular, relations between process steps need to be explicitly visualized, i.e., the relation of a process step (e.g., an x-ray examination) to its preceding and subsequent steps (e.g., a knee surgery). Moreover, every process step has to be associated with additional information, e.g., on the role executing the next step. A ward, for instance, should have information on the type of surgery in order to be able to adequately prepare the patient.

\section{3) Problem Area 3 - Access to patient information:}

Enterprise Viewpoint: Due to paper-based medical records, both the access to patient information (e.g., findings from an x-ray examination) and the retrieval of needed information (e.g., on medical problems of the patient) constitute difficult and time-consuming tasks from the enterprise viewpoint. Figure 3 shows that the participants disagreed with the statement that needed information is displayed at a glance. This, in turn, results in another problem. If information is not visualized at a glance, users must search for currently needed process information. Figure 4 summarizes answers on the question whether important process information can be quickly found. As can be seen, the median is neither agree nor disagree, but the scale is much bigger. Taking into account interview results, this phenomenon can be explained as follows: all participants argued that finding information quickly is easier for experienced staff. New employees, in turn, confirmed having difficulties with finding the needed process information in time.

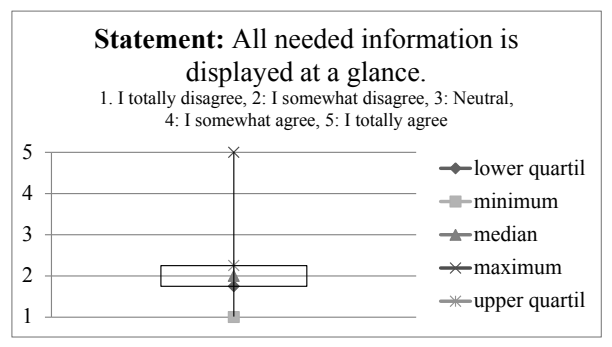

Figure 3. Handling of information 1

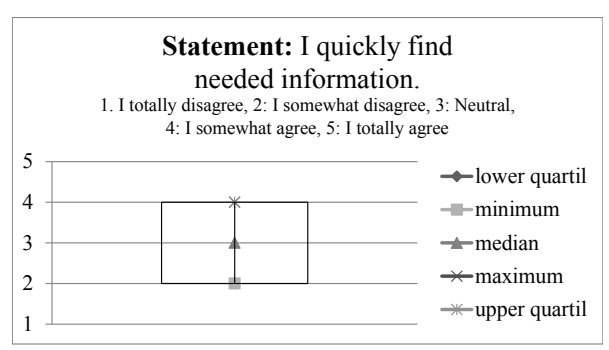

Figure 4. Handling of information 2

From this we derive the most important requirement from the enterprise viewpoint: The need for information containers, each providing all process information needed for performing a particular process step (R6). Based on them centralized access to and management of process information becomes possible.

User Viewpoint: From the user viewpoint access to the medical record in the context of a treatment process is limited as there exist only one instance of a patient's medical record. In the worst case, this paper folder gets lost. This leads, in turn, to a massive financial damage for the hospital since invoicing (i.e., a specific task to be accomplished at the end of any treatment process) is based on the record's content. In such a case, the controlling department has to manually check for the completeness of the medical record. Additional difficulties stem from the fact that many process information are rather unstructured. In the hospital where we conducted the interviews the medical record, for example, consists of numerous paper sheets in random order.

As a consequence, the most important requirements from the user viewpoint are that a cross-departmental, harmonized access to process information needs to be ensured (R7) and moreover, that a consistent information order is required (R8) (e.g., a chronological order). This gives users orientation, while searching for process information. It further improves fast access to needed 
process information.

Presentation Viewpoint: From R6 it becomes evident that an adequate visualization of process information (PR4) is a major requirement from the presentation viewpoint. Visualization has to provide the user with an overview of all available information depending on the current process context. For example this helps the controlling department to simplify completeness checks of the medical record and thus the whole invoicing.

Based on R7 and R8, process information must be explicitly linkable to single process steps along the process (PR5). This facilitates the user's understanding of information and improves user orientation. Thereby, the user should be able to adjust the granularity level of visualized information, for example, if detailed information to single process steps is needed, or just an abstract description of the entire process.

\section{Case Study 2: Automotive Domain}

Our second case study was conducted at a company operating in the automotive sector. Nine interviews with seven knowledge-workers and two decision-makers were conducted. Like in the first case study, the participants were selected based on the premise that typical classes of users dealing with process information are involved.

Again, we identified three major PAs:

\section{1) Problem Area 4 - Roles:}

Enterprise Viewpoint: From the enterprise viewpoint one major problem is the insufficient definition of roles when executing processes. On the one hand, roles are often not completely defined (as important information on tasks and responsibilities is missing). The role "process owner" was mentioned as being a typical example of incomplete defined roles by the interviewees. According to its definition, this role is responsible for a whole process. In practice, however, different people are responsible for different process steps, i.e., the definition is hard to enforce. On the other hand, role definitions are not consistently used across departmental borders. Hence, the requirement roles should be completely defined and role profiles be generally accepted (R9) results from the enterprise viewpoint.

User Viewpoint: From the user viewpoint definitions of roles do not help in many situations anyway (even if we assume that roles are completely defined). As example consider an employee when being confronted with a specific problem during the execution of a process step. In such situation our case study participants typically contact competent staff (in the role of an "expert"), but do not look for solutions in guidelines or manuals. However, finding these "experts" can be difficult in practice, because process steps and appropriate contact persons are not linked. Consequently, the most important requirement from the user viewpoint is that process steps must be linked with associated roles (R10) in order to guarantee fast access to contact persons along the process.

Presentation Viewpoint: From the presentation viewpoint information on contact persons (telephone number or e-mail address) should be adequately visualized (PR6). Also, it should be possible to connect multiple roles to a process step to allow for a finer differentiation of contacts.

\section{2) Problem Area 5 - Access to process information:}

Enterprise Viewpoint: From the enterprise viewpoint interview participants argued that too much information is available (cf. Fig. 5). Five out of nine participants rated the amount of available information as "too high". The interviews showed that documents are distributed over many sources, such as shared drives, databases, or e-mails. Additionally, five out of nine participants "totally disagreed" that needed information is displayed at a glance (cf. Fig 6). One reason are inconsistent folder structures of shared drives across different departments, another one concern the missing availability of an appropriate search function to deal with distributed process information.

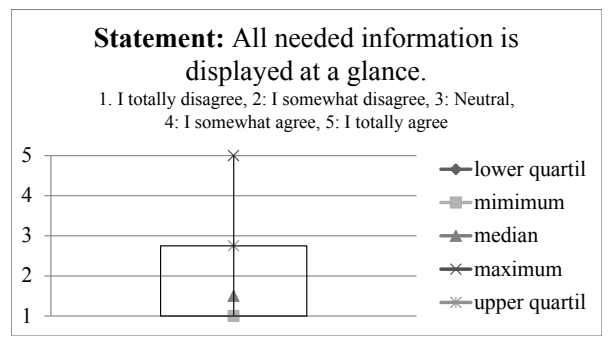

Figure 5. Handling of information 3

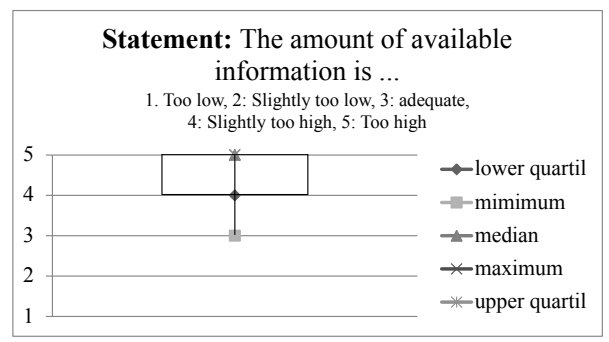

Figure 6. Handling of information 4

As a consequence, the most important requirement resulting from the enterprise viewpoint is that available information must be automatically converted to process information (R11) to reduced information overload. The user should have access to relevant process information on the level of detail he currently needs. As example consider a multipage document. If only one section includes the needed information, the other sections should be automatically dismissed beforehand to only provide relevant process information to the users. 
User Viewpoint: From the user viewpoint participants argued that they do not get process information at an appropriate granularity level. Consider a decision-maker and a knowledge-worker dealing with the same process step (e.g., the task to modify a control unit software). The knowledgeworker stated the need for detailed process information to successfully work on this process step, (e.g., on the guidelines, checklists or tools he has to use). Manager, in turn, are interested in the fact whether or not the process step has been already finished. Participants argued that a contextdependent provision of process information is missing in practice.

To avoid data overload and to enable fast access to personalized process information, the most important requirement from the user viewpoint is that process information must be provided dependent on the user's role (R12).

Presentation Viewpoint: From the presentation viewpoint relations between personalized process information and single process steps must be visualized along the entire process (PR7) to give the user a better orientation and overview. This follows from R11 and R12. Process information needs to be clustered and aggregated according to both user role and current process step. Thereby, different granularity levels for different roles can be graphically represented by the zoom factor on the process. The visualization should be configurable for single users to satisfy personal needs.

\section{3) Problem Area 6 - Search for process information:}

Enterprise Viewpoint: Two search approaches are typically used in the company where we conducted the interviews. The search function provided by the operating system and the built-in search function of an existing enterprise portal. From the enterprise viewpoint this results in several problems. Participants argued, for example, that the search function of the operating system has insufficient search options concerning process steps and roles. Therefore, fulltext search options are often used. However, full-text search often takes too long and thus prevents fast access to needed information.

The built-in search function of the information portal, in turn, provides additional search options (e.g., for information type or categories), but results are only based on a very limited amount of information. In fact, only information hardwired in the portal is included. Further, most participants argued that needed information often cannot be accessed due to missing access rights on shared drives hosting the linked process information.

Consequently, the most important requirement from the enterprise viewpoint is that a search function providing additional retrieval options concerning specific process steps (R13) is needed. In particular, it could help users to refine search results to their personal needs.

User Viewpoint: From the user viewpoint participants argued that search results are limited to (office) documents. Other information, e.g., on roles associated with documents, is missing. Generally, participants confirmed that they often search for process information in the context of their daily work. Problem is, however, that needed information has to be manually extracted from (often large) documents. This is time-consuming and can lead to a misinterpretation.

Therefore, the automated extraction of process information from search results (R14) is required. Search results should only comprise relevant process information depending on the current process context. This is the most important requirement resulting from the user viewpoint.

Presentation Viewpoint: To meet the requirements from the enterprise and user viewpoint (R13 and R14) the presentation of search results is critical. In particular, search results must be adequately visualized (PR8), e.g., in an appropriate sequence constructed based on different, context-sensitive relevance criteria.

\section{Conclusion}

In total, we identified six problem areas (PAs) in our exploratory case studies. We analyzed them following three different viewpoints. Following these viewpoints we can identify first requirements on the effective and efficient handling of process information in an enterprise context. Note that the overall number of requirements can be higher as additional sources for requirements will be continuously considered such as literature studies, practical field work or additional case studies. Figure 7 summarizes our identified requirements.

\section{OnLINE SURVEY}

To further investigate our case study results, we performed an online survey. Due to space limitations we can only present some results here, but a technical report will be published summarizing all results. 219 people (73\% male, $27 \%$ female) from more than 100 different companies participated. The majority of them $(96 \%)$ works in Germany. $57 \%$ of all participants are knowledge-workers, $26 \%$ are managers and $17 \%$ provide no information about their position.

First, we asked participants about the benefits of a process information portal (cf. Fig. 8, Statement 1). $85.85 \%$ of them totally or somewhat agree that central access to process information would help them in their daily work (cf. R7 in Sec. III-B3). More specifically, $18.72 \%$ totally agree that step-by-step guidance (regarding past, current and future process steps) would help them (cf. Fig. 8, Statement 2). $39.66 \%$ somewhat agree with that statement (cf. PR5 in Sec. III-B2). 


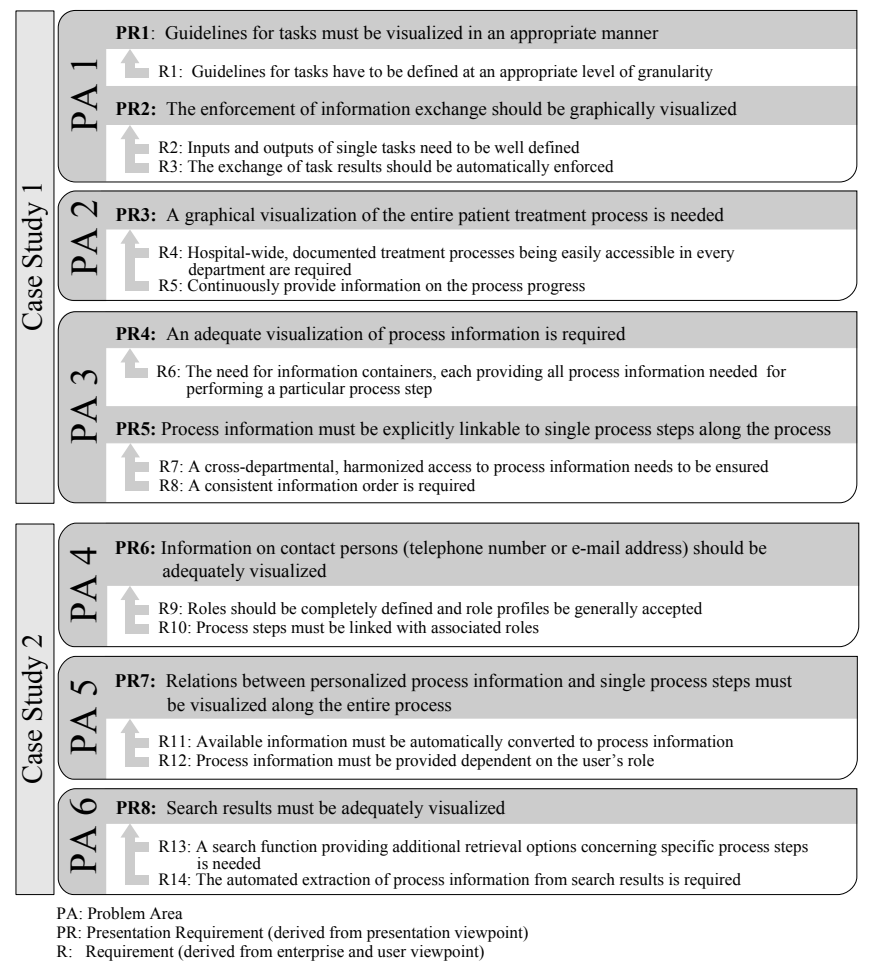

Figure 7. Requirements on the handling of process information.

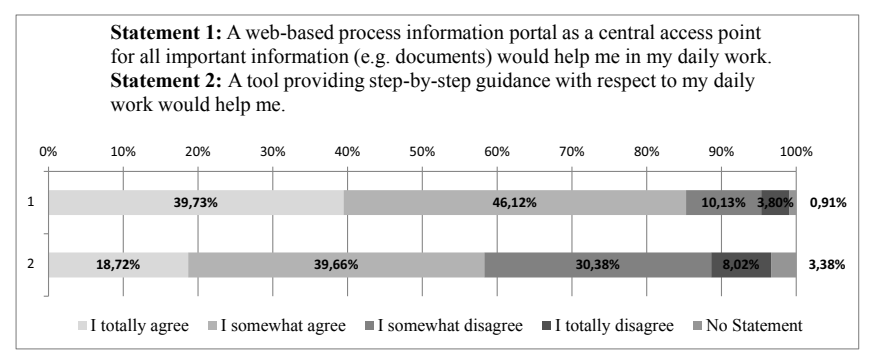

Figure 8. Online Survey Results 1.

Second, we addressed the context-sensitive provision of process information. As depicted in Figure 9 (Statement 1) the majority of respondents $(76.71 \%)$ totally or somewhat agree with the statement that it would be helpful to automatically get relevant information depending on the current process context (cf. R5, PR5, PR7). Only 5.48\% totally disagree. We also ask for the relevance of a continuously available process overview (cf. Fig. 9, Statement 2). 30.59\% totally agree that such an overview would be helpful. $42.92 \%$ somewhat agree (cf. R4, PR3).

Finally, we ask for user preferences when retrieving information. In our case study interviews, the use of search function is often mentioned. Our online survey (cf. Fig. 10) confirms this. Specifically, we ask for the most common way to retrieve information. While $40.18 \%$ of the respondents use search functions, $40.65 \%$ of them prefer navigating along existing structures (e.g., along folder structures in file explorers) (cf. R13, R14, PR8).

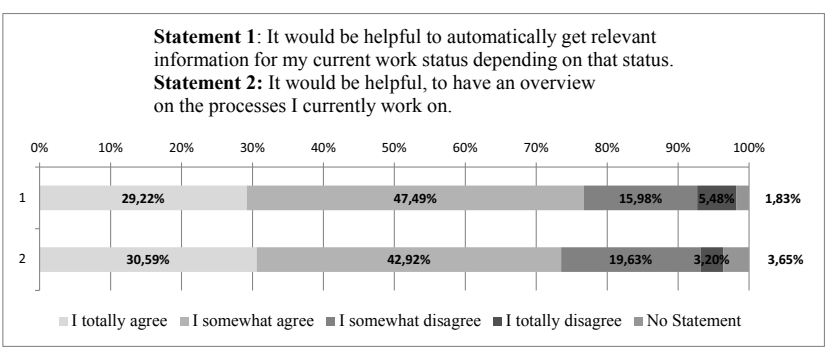

Figure 9. Online Survey Results 2.

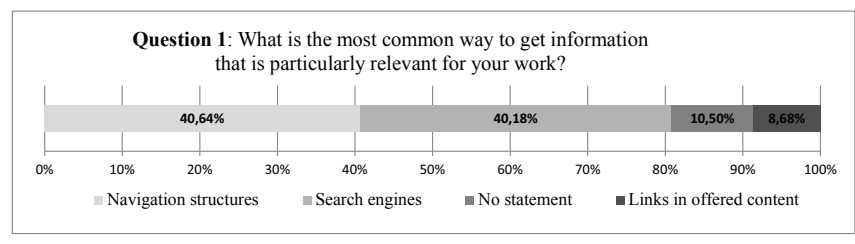

Figure 10. Online Survey Results 3.

\section{A. Conclusion}

The online survey confirms many case study results. In particular, that providing process information in a contextsensitive manner is required. The majority of respondents state that they would benefit from a process information portal (cf. Sec. II-A).

\section{RELATED WORK}

There only exists few research that specifically deals with the handling of process information from a user perspective. Dinter and Winter [15], for example, analyse current information logistics strategy practices by means of a survey. Their findings show that information logistics strategy is linked to company size and governance type. Bucher and Dinter [16] suggest supporting and improving an organizations' operational processes by embedding analytic information into the execution of the processes. Their work provides insights into the dominant design factors and realization approaches of process-oriented information logistics. Finally, van der Heijden [17] investigates success factors for presenting information to end users. He shows that visual attractiveness of information is significantly influenced by its usefulness and ease-of-use.

Further there are research approaches in the field of process visualization. An approach for visualizing eventdriven process chains is presented in [18]. In [19] and [20] an approach for embedding process visualizations in larger enterprise architecture models is presented. [21] describes an approach for a qualitative visualization of processes. A recent approach is provided by the PROVIADO framework [22], [23], which enables the flexible, configurable visualization of complex processes. A view mechanism allows for the adaptation of process models depending on the users' needs, e.g., by reducing the amount of displayed process elements based on well defined view operations. A template 
mechanism task enables the support of different graphical process notations.

\section{SUMMARY AND OUTLOOK}

This paper discusses the need of a personalized, contextaware delivery of process information. Today's data overload makes it difficult to provide knowledge-workers and decision-makers with the needed information at the right time and in the right format and granularity. Picking up this need, this paper presents results from three empirical studies: two exploratory case studies from the automotive domain and the healthcare sector, and an online survey with 219 participants. We identify and describe problems with respect to process-oriented information management in general and the personalized provision of process information in particular. Thereby, we adopt a strict end user perspective. Based on the results of the three empirical studies, we derive first requirements on the user-adequate handling of process information.

Based on the identified requirements, we will develop innovative process visualization concepts as well as interactive process navigation approaches to support the introduction of intelligent, user-friendly process information portals.

\section{REFERENCES}

[1] D. Müller, J. Herbst, M. Hammori, and M. Reichert, "IT support for release management processes in the automotive industry," in 4th Int'l Conf. on Business Process Management (BPM'06), Vienna, Austria, Springer, pp. 368-377, 2006.

[2] R. Lenz and M. Reichert, "IT support for healthcare processes - premises, challenges, perspectives." Data and Knowledge Engineering, Elsevier. Vol. 61, No. 1, pp. 39-58, 2007.

[3] B. Mutschler, M. Reichert, and J.Bumiller, Unleashing the Effectiveness of Process-oriented Information Systems: Problem Analysis, Critical Success Factors and Implications. IEEE Transactions on Systems, Man, and Cybernetics (SMC) - Part C: Applications \& Reviews, pp. 280-291, 2008.

[4] M. Havey, Essential Business Process Modeling. O'Reilly Media, 2005.

[5] B. Weber, M. Reichert, W. Wild, and S. Rinderle-Ma, Providing Integrated Life Cycle Support in Process-Aware Information Systems. in: Int'1 Journal of Cooperative Information Systems (IJCIS) , 18(1), pp. 115-165, 2009.

[6] M. Reichert, S. Rinderle-Ma, and P. Dadam, Flexibility in Process-aware Information Systems. LNCS Transactions on Petri Nets and Other Models of Concurrency (ToPNoC), Special Issue on Concurrency in Process-aware Information Systems. , LNCS 5460, Springer. Vol. 2, pp. 115-135, 2009.

[7] J. Mendling, Metrics for Process Models: Empirical Foundations of Verification, Error Prediction, and Guidelines for Correctness. Springer, 2008.

[8] U. Meissen, S. Pfennigschmidt, A. Voisard, and T. Wahnfried, "Context- and situation-awareness in information logistics," in Current Trends in Database Technology - EDBT 2004 Workshops, pp. 335-344, 2005.
[9] T. Petrocelli, Data Protection and Information Lifecycle Management. Prentice Hall, 2005.

[10] M. J. D. Sutton, Document Management for the Enterprise: Principles, Techniques, and Applications. Wiley, 1996.

[11] K. Reichenberger, Kompendium semantische Netze: Konzepte, Technologie, Modellierung (X.media.press) (German Edition). Springer, 2010.

[12] D. J. Mayhew, The Usability Engineering Lifecycle: A Practitioner's Handbook for User Interface Design (Interactive Technologies). Morgan Kaufmann, 1999.

[13] A. Syed and A. Shah, "Data, information, knowledge, wisdom: A doubly linked chain?" in Proc. of the 2006 Int'l Conf. on Information \& Knowledge Engineering, Las Vegas, USA, pp. 270-278, H. R. Arabnia and R. R. Hashemi, Eds., 2006.

[14] J. Rowley, "The wisdom hierarchy: representations of the DIKW hierarchy," J. Information Science 33 (2), pp. 163 180, 2007.

[15] B. Dinter and R. Winter, "Information logistics strategy analysis of current practices and proposal of a framework," in Proc of the 42nd Hawaii Int'l Conf on Sys Sciences, pp. 1 $-10,2009$

[16] T. Bucher and B. Dinter, "Process orientation of information logistics - an empirical analysis to assess benefits, design factors, and realization approaches," in Proc of the 41st Hawaii Int'l Conf on Sys Sciences, p. 392. IEEE Computer Society, 2008.

[17] H. van der Heijden, "Factors influencing the usage of websites: the case of a generic portal in the netherlands," Information \& Management Vol 4, pp. 541 - 549, 2003.

[18] J. Mendling, A. Brabenetz, and G. Neumann, EPML2SVG - Generating Websites from EPML Processes. in: Proc. $3^{\text {rd }}$ GI Workshop on Event-Driven Process Chains (EPK'04), Luxembourg, pp. 55-64, 2004.

[19] M. Steen, D. Akehurst, H. t. Doest, and M. Lankhorst, Supporting Viewpoint-Oriented Enterprise Architecture. in: Proc. $8^{\text {th }}$ IEEE Int'l. Enterprise Distributed Object Computing Conference (EDOC'04), pp. 201-211, Monterey, 2004.

[20] S. Buckl, A. M. Ernst, J. Lankes, C. M. Schweda, and A. Wittenburg, Generating Visualizations of Enterprise Architectures using Model Transformations. in: Proc. Enterprise Modelling and Information Systems Architectures (EMISA'07), pp. 3346, 2007.

[21] K. Wittenburg and L. Weitzman, Qualitative Visualization of Processes: Attributed Graph Layout and Focusing Techniques. in: Proc. of the Symposium on Graph Drawing (GD'96), LNCS 1190, pp. 401-408, London, 1996.

[22] R. Bobrik, M. Reichert, and T. Bauer, "View-based process visualization," in 5th Int'l Conf. on Business Process Management (BPM'07), September 2007, Brisbane, Australia, Springer, pp. 88-95, 2007.

[23] R. Bobrik, T. Bauer, and M. Reichert, "Proviado - personalized and configurable visualizations of business processes," in Proc. 7th Int'l Conf. on Electronic Commerce and Web Technologies (EC-WEB'06), Krakow, Poland, Springer, pp. 61-71, 2006. 